\title{
Two-dimensional domain structures in Lithium Niobate via domain inversion with ultrafast light
}

\author{
X. Chen, ${ }^{1}$ P. Karpinski, ${ }^{1,2}$ V. Shvedov, ${ }^{1}$ B. Wang, ${ }^{3}$ J. Trull, ${ }^{3}$ C. Cojocaru, ${ }^{3}$ A. Boes, ${ }^{4}$ A. Mitchell, ${ }^{4}$ W. \\ Krolikowski, ${ }^{1,5}$ and Y. Sheng ${ }^{1, *}$ \\ ${ }^{1}$ Laser Physics Centre, Research School of Physics and Engineering, Australian National University, Canberra, \\ ACT 0200, Australia \\ ${ }^{2}$ Wroclaw University of Technology, Wybrzeze Wyspianskiego, Wroclaw, Poland \\ ${ }^{3}$ Univeristat Politecnica de Catalunya, Terrassa, Barcelona, Spain \\ ${ }^{4}$ ARC Centre of Excellence, CUDOS, School of Electrical and Computer Engineering, RMIT University, Melbourne, \\ VIC 3001, Australia \\ ${ }^{5}$ Science Program, Texas A\&M University at Qatar, Doha, Qatar
}

Received May 31, 2016; accepted June 27, 2016; published June 30, 2016

\begin{abstract}
Periodic inversion of ferroelectric domains is realized in a lithium niobate crystal by focused femtosecond near-infrared laser beam. One and two-dimensional domain patterns are fabricated. Quasiphase matched frequency doubling of $815 \mathrm{~nm}$ light is demonstrated in a channel waveguide with an inscribed periodic domain pattern with conversion efficiency as high as $17.45 \%$.
\end{abstract}

Lithium niobate $\left(\mathrm{LiNbO}_{3}\right)$ waveguides play a significant role in integrating many optical devices due to excellent electro-optic, acousto-optic, and nonlinear properties of this important material. In nonlinear interactions such as frequency conversion the phases of interacting waves can be synchronized in $\mathrm{LiNbO}_{3}$ waveguide through the process of quasi-phase matching (QPM) [1], which requires a periodic ferroelectric domain inversion to change the sign of the second-order nonlinear susceptibility at every coherence length. A common method used for ferroelectric domain engineering is electric field poling [2], where a spatially modulated electric field is applied along the polar axis of the crystal. This method involves a photolithographic process to fabricate periodic electrodes and application of sequence of high voltage pulses to induce ferroelectric domain flipping. Apart from its complexity, this technique suffers from the restrictions imposed by the crystallographic orientation of the crystal. Consequently, while the Z-cut crystals are easily poled, other orientations require sophisticated design of electrodes and involve etching of the crystal [3].

There have been considerable efforts in developing alternative methods of ferroelectric domain reversal to overcome the drawbacks of traditional electric field poling. In particular, the optical poling uses intense laser

\footnotetext{
* E-mail: yan.sheng@anu.edu.au
}

irradiation to either assist or directly induce domain inversion [4]. This approach becomes particularly interesting because of its unique advantages. For example, it overcomes the restriction that the electric field must be applied along the polar axis of the crystal. In addition, the light field can be manipulated more accurately with a resolution up to the diffraction limit. Therefore, it enables one to fabricate fine ferroelectric domains with better defined details than those produced by electric-field poling alone. The UV radiations are usually used in direct laser poling as their strong absorption by ferroelectric can produce a very high local temperature gradient for domain inversion. However, such a strong absorption of UV light restricts inverted domains into a shallow surface layer (few hundred nanometers). This severely limits the application of such an optically created domain pattern. So far no report has been made on the performance of these structures as a QPM frequency convertor.

In this work we demonstrate that the inversion of ferroelectric domains can be efficiently realized by using a tightly focused femtosecond infrared laser inside the $\mathrm{LiNbO}_{3}$ crystal without application of any electric field. As the $\mathrm{LiNbO}_{3}$ is transparent in the infrared, we can form ferroelectric domain patterns not only close to the surface but also deep inside the medium. We report on the fabrication of a variety of two-dimensional domain structures extending up to 60 microns deep inside the crystal. We used this domain inversion technique to fabricate a periodic domain pattern inside the channel waveguide and demonstrate efficient $(17.45 \%)$ frequency doubling of $815 \mathrm{~nm}$ light.

The experimental setup of the laser poling is shown in Fig. 1(a). We used a Z-cut $500 \mu \mathrm{m}$-thick congruent lithium niobate wafer. The infrared light was generated by a femtosecond oscillator (MIRA, Coherent) operating at $800 \mathrm{~nm}$, with a pulse duration of $180 \mathrm{fs}$ and a repetition 
rate of $76 \mathrm{MHz}$. The light was focused inside the sample by a microscope objective $(\mathrm{NA}=0.65)$ and then scanned transversely to produce the desired domain pattern. The resulting focus spot is about $1.5 \mu \mathrm{m}$ in diameter. The beam was incident normally to the surface of the crystal. The beam was initially focused on the front $(-Z)$ surface of the crystal. Then the sample was translated along the Zdirection so that the position of the focal region moved from the $-\mathrm{Z}$ toward the $+\mathrm{Z}$-surface with an average speed of $v=10 \mu \mathrm{m} / \mathrm{s}$ [5]. Figure 1(b) illustrates the physical principle of our in situ diagnostic of the domain inversion process. It is based on the emission of a transverse second harmonic beam, the so called Cerenkov beam. This emission takes place only when the fundamental beam illuminates the region of spatial modulation of the second order nonlinearity such as the domain wall, which separates regions of positive and negative $\chi^{(2)}$. When the fundamental beam is focused in the uniform region (constant nonlinearity) no Cerenkov signal is emitted. Therefore, the appearance of such a signal during the domain writing process indicates a local domain inversion. Figure 2(a-c) depicts typical images of the resulting infrared optically poled domain patterns after 5 minutes of etching in hydrofluoric (HF) acid. Here three different 2-dimensional domain patterns are displayed: (a) square lattice; (b) hexagonal lattice and (c) parallel lines of domains. These images have been recorded via traditional optical microscopy after etching the sample in HF solution. For comparison, graphs in Fig. 2 (d, e) depict two and three-dimensional visualization of a square domain pattern obtained via a nondestructive Cerenkov second harmonic generation technique. While in Fig.2 (e) only the first 30 microns of the domain pattern are shown, we confirmed that our method allowed us to fabricate domain structures extending up to 60 micrometers inside the $\mathrm{LiNbO}_{3}$ crystal.

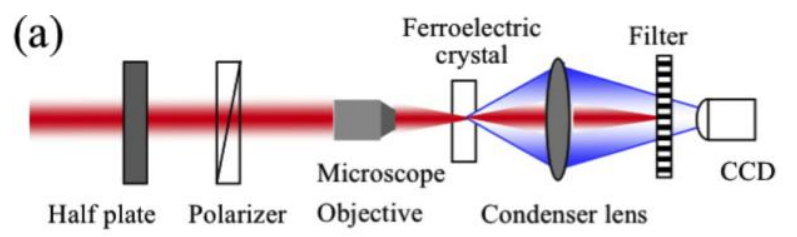

(b)

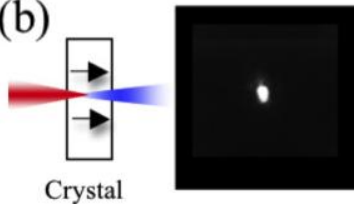

Fig. 1. Experimental setup (a) for femtosecond laser optical poling and in situ monitoring (b-c) of ferroelectric domain inversion via

Čerenkov-type second harmonic scanning microscopy. (b) Only forward second harmonic is emitted when the fundamental beam is focused in uniform crystal. (c) transverse Cerenkov second harmonic is emitted when the fundamental beam illuminates ferroelectric domain wall.
We believe that our femtosecond laser poling shares the same underlying physical mechanism as the UV light poling. The presence of a thermoelectric or/and pyroelectric field in the focal volume of the beam acts as a possible cause of domain inversion. As $\mathrm{LiNbO}_{3}$ is transparent in the infrared, the multi-photon absorption of high intensity light would heat the crystal in the focal area. While the used $800 \mathrm{~nm}$ is too long for band to band two photon absorption (the band gap of LiNbO3 is $\sim 4 \mathrm{eV}$ ), the process could involve two or higher order photon absorption as well as defects or impurity states within the gap.
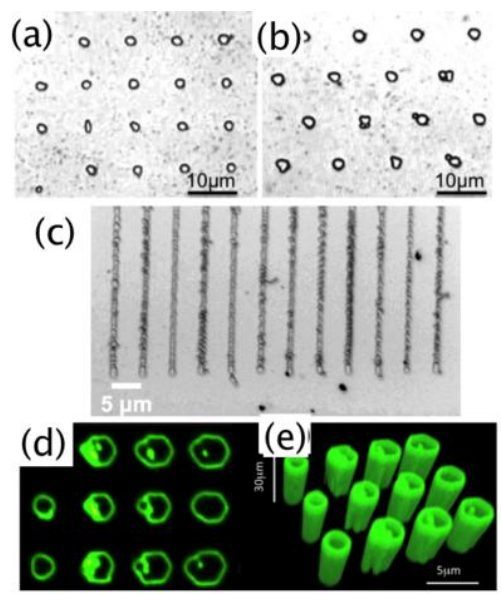

Fig. 2. (a-c) Microscopic images of all-optically fabricated twodimensional domain patterns. (h,i) visualization of square domain pattern with Cerenkov microscopy.

As a further demonstration of our infrared optical poling technology, the ferroelectric domain engineered $\mathrm{LiNbO}_{3}$ waveguide was used to generate quasi-phase matched second harmonic [7]. The Ti-indiffused $\mathrm{LiNbO}_{3}$ channel wave-guide was formed on the $-Z$ surface of a $500 \mu \mathrm{m}$ thick congruent crystal. It was fabricated by diffusing a $35 \mathrm{~nm}$ thick Ti stripe with a width of $3 \mu \mathrm{m}$ into the crystal surface, using a diffusion time of $22 \mathrm{~h}$ and a diffusion temperature of $1010^{\circ} \mathrm{C}$. The $\mathrm{Ti}$ waveguide was designed to support a single mode at the non-linear optical pump wavelength of $815 \mathrm{~nm}$, with a refractive index contrast of approximately $\Delta \mathrm{n}=0.001$ and a mode depth of $\sim 3 \mu \mathrm{m}$. The loss of the waveguide was measured to be around $0.1 \mathrm{~dB} / \mathrm{cm}$ for both fundamental and second-harmonic TM modes. The quasi phase matching period is $2.74 \mu \mathrm{m}$, aimed at frequency doubling of $815 \mathrm{~nm}$ light. We used $10 \mathrm{~mm}$ long waveguides with a periodic domain pattern covering the whole length as seen in Fig. $3(\mathrm{a}, \mathrm{b})$. Prior to the nonlinear optical measurements, the waveguides with inscribed domains were annealed for $30 \mathrm{~min}$ at $200^{\circ} \mathrm{C}$ to remove any residues of photorefractive effect. We used a $\mathrm{NA}=0.1$ microscopic objective to focus the $815 \mathrm{~nm}$ laser beam from the femtosecond oscillator (MIRA, Coherent) into the waveguide and collected the emitted second 
harmonic using a $\mathrm{NA}=0.2$ microscopic objective. It should be stressed that the losses introduced by the femtosecond poling were negligible. Temperature tuning was used to optimize the frequency doubling process and the maximum harmonic output was achieved at $62.5^{\circ} \mathrm{C}$. Figure 3(c) shows the dependence of the power of output second harmonic on the input power of a fundamental wave. For low input power, the power of second harmonics follows the square law dependence. The growth slows down above $90 \mathrm{~mW}$ of input power. A second harmonic power of $26.17 \mathrm{~mW}$ was obtained for $150 \mathrm{~mW}$ of input power. This gives conversion efficiency of $17.45 \%$ and the normalized conversion efficiency of $199.3 \% \mathrm{~W}^{-1} \mathrm{~cm}^{-2}$.
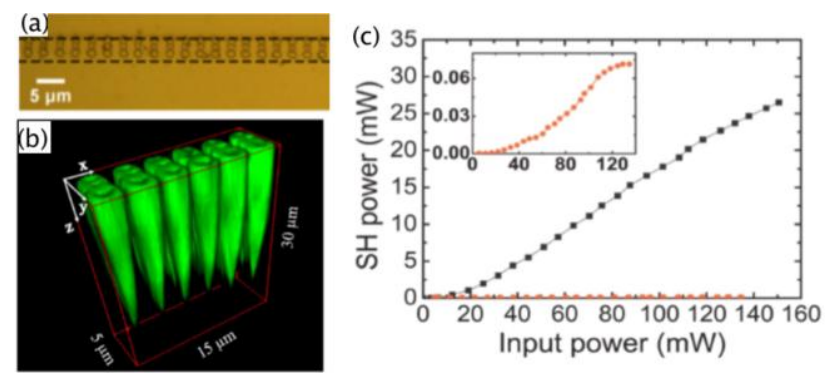

Fig. 3 Quasi-phase matched grating in Lithium Niobate waveguide. (a) Optical microscopic image of the 2-D optically poled domain pattern with the period of $2.74 \mu \mathrm{m}$ in the $\mathrm{X}$ direction and $1.15 \mu \mathrm{m}$ in the $\mathrm{Y}$ direction. Individual inverted domains are visible as small circles. Waveguide boundaries are indicated with dashed lines. (b) Threedimensional profiles of the inverted domains obtained by the Cerenkov second-harmonic microscopy [6]. (c) Average power of the second harmonic versus the average power of the fundamental wave at the optimal quasi-phase matching temperature $62.5^{\circ} \mathrm{C}$. The black squares and red dots represent the results of quasi-phase matched and pure waveguides without poling, respectively.

In conclusion, we have demonstrated all-optical fabrication of quasi-phase matched structures based on ferroelectric domain inversion in $\mathrm{LiNbO}_{3}$ wafers and waveguides using femtosecond infrared pulses. The proposed scheme allows one to realize an efficient quasiphase matching using the highest modulation depth of nonlinearity from $\chi^{(2)}$ to $-\chi^{(2)}$, without introducing propagation loss for interacting waves. Conversion efficiency of $17.45 \%$ is measured for the second-harmonic generation in a $10 \mathrm{~mm}$ long domain inverted pattern inside a Titanium in-diffused channel waveguide. Our results indicate that the infrared laser poling constitutes a powerful method for fabricating periodic ferroelectric domains in an all-optical manner, thereby allowing for a wealth of new possibilities for precise and flexible domain engineering.

This work was supported by the Australian Research Council (ARC); Qatar National Research Fund (NPRP 8-
246-1-060). X. Chen acknowledges support from the China Scholarship Council for his Ph.D. Scholarship No. 201306750005. P. Karpinski thanks the Polish Ministry of Science and Higher Education for the "Mobility Plus" scholarship.

\section{References}

[1] J. A. Armstrong, N. Bloembergen, J. Ducuing, P.S. Pershan, Phys. Rev. 127, 1918 (1962).

[2] M. Houe, P.D. Townsend, J. Phys. D 28, 1747 (1995).

[3] Y. Sheng, T. Wang, B. Ma, E Qu, B. Cheng, D. Zhang, Appl. Phys. Lett. 88, 041121 (2006).

[4] C.Y.J. Ying, A.C. Muir, C.E. Vakduvuam G.H. Steigerwald, C.L. Sones, R.W. Eason, E. Soergel, S. Mailis, Laser Photonics Rev. 6, 526 (2012).

[5] X. Chen, P. Karpinski, V. Shvedov, K. Koynov, B. Wang, J. Trull, C. Cojocaru, W. Krolikowski, Y. Sheng, Appl. Phys. Lett. 107, 141102 (2015).

[6] Y. Sheng, A. Best, H. Butt, W. Krolikowski, A. Arie, K. Koynov, Opt. Expr. 16, 16539 (2010)

[7] X. Chen, P. Karpinski, V. Shvedov, A. Boes, A. Mitchell , W. Krolikowski, Y. Sheng, Opt. Lett. 41, 2410 (2016). 